\title{
An Exploratory Study on Consumers' Attention towards Social Media Advertising: An Electroencephalography Approach
}

\author{
Hui-Chih Wang \\ National Taiwan University of Science and Technology \\ hcwang1@gmail.com
}

\author{
Her-Sen Doong \\ National Chiayi University \\ hsdoong@mail.ncyu.edu.tw
}

\begin{abstract}
This study aimed to demonstrate empirically that consumers' attention is related to changes in the electroencephalographic (EEG) brain activity during the observation of a humorous or non-humorous advertisement displayed on social media. This study is innovative in three ways: first, social media is a popular platform for the testing of pre-launch advertisements. However, the online environment makes concealing real thoughts easy. Hence, the skill of observing consumers' actual responses to advertising via brain activity has useful implications. Second, by examining how men and women differ neurologically in sensing humor, this study is a pioneer in testing the interaction effects from an EEG signal perspective. Third, by using fuzzy set qualitative comparative analysis ( $s Q C A$ ) methods rather than traditional means, such as correlation, a new lens for understanding neuromarketing might be built.
\end{abstract}

\section{Introduction}

Neuromarketing, which applies neuroscience knowledge to assist the understanding of consumer responses, is a pioneering research field. This study adds value to the online social media research field by identifying an opportunity where neuroscientific observation can uncover how males and females differ in their attention towards humor-based social media advertising from an electroencephalograph (EEG) perspective.

As businesses tend to believe that "where the eye stops, the sale begins" [38], the challenge for professionals is to hold consumers' attention via advertising. Such a goal is even more difficult today for social media advertisers due to the overwhelming number of advertisements online which compete with each other to attract consumers. Practitioners found humor a useful communication tactic to deal with this problem [11]. For example, in 2013, in the United States alone, spending on advertising is reported to have been US\$157.2 billion, and $10-30 \%$ of the total number of advertisements were intended to be humorous [27].

While there is a growing interest in discussing how humor might enhance consumers' engagement in online social networks [21] and on social media [33], the effect that gender differences may have on consumers' responses towards social media is also becoming a concern of scholars. For example, Lim et al. [29] reported that females consistently showed higher social media site satisfaction and loyalty than did males. Chan et al. [7] surveyed 221 experienced Facebook users and reported that while men perceived the entertainment a more crucial factor shaping their satisfaction, women regarded the relationship maintained a more important issue affecting their satisfaction. However, whether men and women differ in their advertising attention on online social media channels is still under investigation. In sum, Porter et al. [39] argued that investigating the gender issue is crucial for online business given that it is a useful and profitable segmentation variable.

Importantly, past studies in the field of social media advertising mainly applied traditional measures, such as questionnaires or interviews. Vecchiato et al. [46], however, argued that subjects might hide their true feelings from interviewers who represent the company while participating in pre-launch research. This issue might also become a challenge for online social media researches. Measuring subjects' real engagement while they are watching advertisements via neurosciencebased techniques may become a useful method. In support of this, Lin et al. [30] indicated that an EEG is a feasible way to characterize individuals' reactions to a stimulus, such as the music or advertising.

The current study is therefore important because it aims to bridge the gaps between a number of existing domains: (1) online research has reported various gender influences on individuals' decision-making and intentions (e.g., [18]). Evidence has reported that such diversity can be found in humor production and comprehension [20]. However, these studies focus mainly on how the gender differences in humor ability could be used in the domain of mate selection. Little is known about how these differences are associated with 
humor advertising strategies and brain activity. (2) Gender is a type of biological diversity. Since the nature of neural data is biological responses, it is important to carefully evaluate the gender impact on Neuromarketing findings [9]. For example, Riedl et al. [40] reported that while processing information about eBay offers, women activate more brain areas compared with men. Nevertheless, while the humor comprehension process via the neuroimaging (fMRI) approach might have been studied already [42], the impact of gender differences has been neglected. (3) Online social media is a powerful advertising channel, and methods to investigate how men and women may differ in their respond to these advertisements are developing (e.g., [24]). Nonetheless, these studies have generally focused on the cognitive approach and have overlooked the lens of brain activity. (4) Unlike past studies, which mainly use the method of multiple regression analysis, the present study applies the fuzzy set qualitative comparative analysis (fsQCA) method, which is new to this discipline, to uncover more insights into EEG brain activity.

In sum, this study aims to create multi-disciplinary knowledge that is beyond the current scope of social media and neuromarketing research. In the following sections, a literature review discusses online social media advertising and consumer attention and gender differences in information processing. Based on these discussions, the research propositions and research model are proposed. The research methodology, data analysis, and conclusion are also presented.

\section{Literature review}

\subsection{Consumers' attention to advertisement}

Advertising is known as a powerful tool to communicate with a large number of consumers and build their knowledge about a specific brand [4, 27]. In the e-marketplace, which lacks the personnel to serve consumers face-to-face, online advertising plays a particularly important role in offering consumers product/service information, persuading them, and facilitating their purchase intentions. Today, online social media like Facebook, after years of rapid growth, are more like a strategy channel for wealth creation and social capital development, rather than a communication medium alone [43]. As more and more companies are using online social media as a key tactic in their marketing practices [22] to promote their product and brand information to potential customers, practitioners are facing new challenges and opportunities: (1) how to use such a channel to publish advertisement; (2) how to evaluate customers' responses to these social media advertisements.
Numerous models have been proposed to delineate how consumers respond to advertisements. These models are often hierarchical in nature. For example, early in 1925, Strong [45] proposed a well-known model in the marketing field, known as the AIDA model; this model suggests that consumers' purchase decisionmaking process will go through four stages, namely, attention, interest, desire, and action. Since then, getting consumers' attention has become an important goal for advertisers, as it initiates customers' communication with the organization.

Today, how consumers act on online social media and how they interact with the business on this channel has received increasing attention. Chu [8] reported that Facebook group members are likely to present a higher level of self-disclosure towards viral advertising responses than are non-group members, but such a tendency will not shape their advertising pass-on behavior. Dao et al. [13] indicated that informativeness, entertainment, and credibility positively affect online customers' perceived value of social media advertising, which ultimately leads to the online purchase intention.

Notably, these existing studies have mainly applied methods such as personal interviews, focus groups, or self-administered questionnaires to investigate consumers' attention toward online advertisements. However, only a handful of studies have documented how brain activity might affect consumers' attention towards the advertisements.

For example, Costa et al. [10], who were interested in interdependency among different brain regions, collected brain activity while subjects were watching emotional or non-emotional film stimuli. Happiness was reported to create widespread synchronization among frontal and occipital sites. Sadness, on the other hand, was found to generate a pattern involving great information exchange among frontal channels.

Some researchers have observed the associations between subjects' EEG activity and their emotions while watching movies [35]. Alpha $(8-12 \mathrm{~Hz})$, beta (13$30 \mathrm{~Hz})$ and gamma $(>30 \mathrm{~Hz})$ activities were found to be more vivid than delta $(1-3 \mathrm{~Hz})$ and theta $(4-7 \mathrm{~Hz})$ activities. By tracking the cortical activity and functional connectivity in normal subjects, a few studies have investigated changes in subjects' brain activity while they watch TV advertisements [36], revealing that the amount of cortical spectral activity from the frontal areas was higher for TV advertisements that were remembered, compared with that stimulated by TV advertisements that were forgotten [2]. Theta activity in the frontal cortical regions was also found to be higher for well-remembered advertisements [25]. These studies have consistently shown that individuals' brain activity is related to the attention paid to the film or advertisement. Consequently, the following is proposed: 
- P1: Differences in EEG brain activity will predict different levels of consumers' attention towards social media advertisements.

\subsection{Gender differences and its business implications}

Gender is like a social resource that is heavily used by advertisers [23] and in our daily life, thousands of advertising images addressing gender lines are around us [48]. For many decades, researchers from a range of disciplines have attempted to delineate the fundamental diversity between men and women (e.g., [49]). These differences are particularly important in advertising and promotion strategies, as gender has long been utilized as a basis for market segmentation. This is because gender meets the requirements for successful implementation that not many other variables are able to meet: (1) men and women are simple to identify, (2) gender segments are easily accessible, given that they are among the top few pieces of data that most retail channels collect, and (3) gender segments are large enough to gain profits [14]. In business practices, both Melnyk et al. [34] and Porter et al. [39] addressed that gender-based segments are useful and profitable.

Upon the acceleration of the e-commerce era, the impact of the gender difference has also attracted increasing attention. For example, compared to men, researchers found that women are more aware of nonverbal social cues in a computer-mediated environment [16] and perceive a greater social presence in online communication [14], and that female bloggers are more sensitive to satisfaction and less responsive to attractive alternatives compared to their male counterparts [50].

2.2.1. Gender differences in social response. Bem [5] argued that men and women use different socially cognitive attributes in information encoding and problem-solving: hence, their perceptions are also diverse. In other words, men and women may take unconscious or internalized actions due to their gender status. Gender diversity is thus an important topic in social behavior research.

For instance, Cross and Madson [12] proposed a review uncovering many gender differences in social experience and behavior. These authors further contended that most social behavior might be better understood if gender differences could be considered independently and interdependently. Many researchers have developed more advanced studies to further clarify this issue. Gabriel and Gardner [17] proposed a model to integrate the relational and collective aspects of interdependence. Following the theory of gender differences in cognition, affect, and behavior, they found that women would focus more on the relational aspects of interdependence, whereas men would focus more on the collective aspects.

Gender effects are also attracting more attention in online social media. Liew et al. [28] surveyed 387 Malaysian adult users and indicated that men and women differ in their adoption-perception-use behaviors on Facebook. Judson et al. [24] surveyed 432 Twitter/Facebook/YouTube users and reported that though men and women portray different levels of selfperceived brand relevance, such links can be changed when social media is examined from the perspective of high/low self-presentation and self-disclosure. Porter et al. [39] examined 232 members from more than 30 firmsponsored virtual communities and reported that men and women presented diverse trust levels towards their trust formation on these social media sites.

\subsubsection{Gender differences from neurobiology aspects.} In the 19th century, researchers initiated investigation into the differences between men's and women's neurobiology. For example, Darwin [15] reported that men's brains are larger than women's. However, more recently, advances in technology have made neuroimaging clearer and more precise. Rushton and Ankney [41] confirmed this notion using magnetic resonance imaging, and further indicated that the average man's cranial capacity is $1,442 \mathrm{~cm} 3$, while that of women is $1,332 \mathrm{~cm} 3$. This finding is important, as Cahill [6] asserted that the size of specific brain aspects may influence decision-making or memory performance. In other words, this might shed light biologically on claims such as "Men are from Mars and Women are from Venus". More recently, Barnett and Cerf [3] applied the cross-brain-correlation (CBC) measure and tested 46 subjects ( 20 women/26 men) who watched trailers and movies at a commercial theater. EEG neural data recording showed that the men and women differed in the film content they engaged with: the women recalled the appearance of a Coca-Cola cup better than did the men while the latter recalled the climactic straw insertion better than the former did. This finding has the interesting implication that in order to communicate with a specific gender segmentation more effectively, the selection of the film content must be chosen strategically.

Specifically, men and women are also biologically different in terms of how they deal with emotional messages. For example, Mak et al. [32] revealed that women's emotion-related brain regions are more active than men's, while in men's brains, the regions related to cognitive information processing are more active than are women's. Consistently, Shirao et al. [44] suggested that men deal with emotional decision-making tasks 
more cognitively rather than emotionally, as in their study, the men's medial prefrontal cortex was significantly activated. In contrast, women's medial prefrontal cortex did not show substantial activation, suggesting that women handle emotional decisionmaking tasks more emotionally. The current study argues that these findings may provide meaningful implications for humor communication in social media advertising.

Humor, in which people enjoy smiling and laughing [1], is related to a strong emotional reaction in the brain via the autonomic and endocrine systems [37]. Humor makes audiences feel happy and relaxed: hence, when selecting mates, women prefer men with a good sense of humor [31]. Similarly, Howrigan and MacDonald [20] revealed that compared to men, women are more emotionally active. Greengross and Miller [19] also contended that humor comprehension is a cognitive process, and men tend to deal with it better than women do. These discussions support the contention that men and women may show diverse responses to humorous/non-humorous advertisements, but none of the past studies have empirically verified this issue from a biological perspective. Consequently, this study proposed the following:

- P2: Men and women will display different of EEG brain activity while watching humorous/nonhumorous social media advertisements.

- P3: The differences in men's and women's EEG brain activity will predict different levels of attention towards humorous/non-humorous social media advertisements.

\subsection{Individuals' changeable biological status}

Most EEG studies, such as Yoto et al. [47] discussed four bands of human brainwave power: delta band $(\mathrm{f}<4$ $\mathrm{Hz})$, theta band $(4 \mathrm{~Hz} \leqq \mathrm{f}<8 \mathrm{~Hz})$, alpha band $(8 \mathrm{~Hz} \leqq \mathrm{f} \leqq$ $13 \mathrm{~Hz})$, and beta band $(13 \mathrm{~Hz}<\mathrm{f} \leqq 30 \mathrm{~Hz})$. The first two are related to sleepiness and the decline in the level of consciousness. While the alpha band is mostly corresponded to the relaxation during the awakening time, the beta band generally reflects a state of excitement or a high level of consciousness.

Figure 2 below demonstrates that after conducting a series of brain activity experiments, this study found that, even for the same person, his/her brain activity is likely to vary at different times during the same day, even while conducting the same activity.

As brain activity reflects a person's current biological condition, such as blood pressure, it varies from time to time even during the same day. The empirical experiences of this study confirmed this.
Figure 2, below, presents four different EEG 3D brain activity recordings for the same subject while he conducted the same activity for one minute at four different times. Before the brain activity recording started, the subject was given one A4 sheet full of 50 easy mathematical questions such as " $10+12=$ ?" Then he was told he had one minute to do these mental calculations and write down the answers. All of the calculations presented were designed to have a similar degree of complexity.
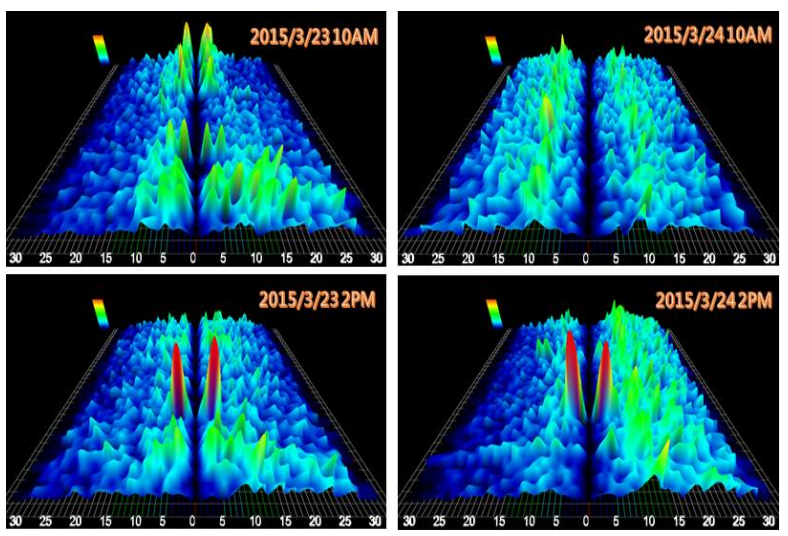

Figure 2. EEG 3D Brain Activity Comparison for the Same Subject

The left line shows the brain activity results of this subject on $2015 / 3 / 23$ at $10 \mathrm{am}$ and $2 \mathrm{pm}$, and on 2015/3/24 10 am and 2 pm, respectively. The middle line of the figure indicates 0 Hertz: the right shows the right frontal brain activity from 1-30 Hertz, and the left shows the left frontal brain activity from 1-30 Hertz. The stronger the specific brain activity, the higher the wave. For example, at 3/23 $10 \mathrm{am}$, high theta activity $(4 \mathrm{~Hz} \leqq \mathrm{f}<8 \mathrm{~Hz})$ could be observed.

Intuitively, we could tell that these four figures showed different patterns of EEG brain activity, which can be observed by the wave shape and height, lightness/darkness, and balance/imbalance of the left and right frontal brain. Figure 3/24 2PM reports very strong right frontal brain activity, while the left frontal brain activity was inactive. Figure 3/24 10 am, however, showed balanced and vivid left/right frontal brain activity. Figure 3/23 10 am revealed a gap during the one-minute mental calculation, and just before the calculation ended, the right frontal brain activity was much stronger than that produced by the left frontal brain.

EEG brain activity is a reflection of how much energy the subject's biological brain can produce. This empirical comparison uncovered the important fact that EEG brain activity is very sensitive to the individual's current biological status. When using this technique to 
assess consumers' responses to commercial advertisements, this fact must be borne in mind and its impact on findings must be carefully evaluated. Nevertheless, past neuromarketing studies have overlooked this important process which is a standard protocol in biology research discipline.

To draw the attention of neuromarketing researchers, this study proposes that a baseline EEG observation shall be considered while collecting the EEG responses of participants who are watching a social media advertisement. As each subject might have a diverse biological brain condition before watching the advertisement, ignoring its impact would create a biased result while interpreting the brainwave results of watching the social media advertisement. Thus, the following was proposed:

- P4: Consumers' EEG baseline brain activity should be evaluated together with their EEG brain activity while watching the social media advertisement.

\section{Research methodology}

Based on the research objectives and propositions, the following research model (Figure 3) was proposed:

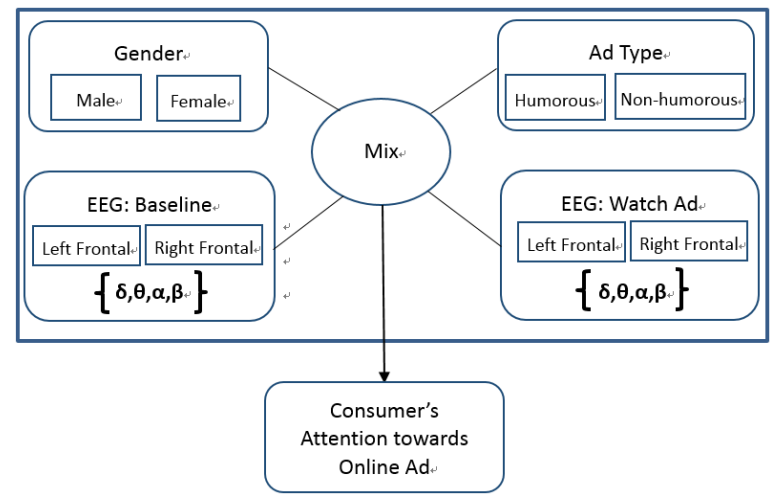

Figure 3 Research Model

\subsection{Subjects}

Thirty-three volunteer subjects aged from 19 to 66 participated in this laboratory experiment. All of them had watched social media advertisements before; hence, they were adequate participants for this study. Subjects were asked to sign an agreement after they had been informed of the overall experimental process and were told that during this process, four electrodes would be placed on their forehead and behind their ears. Subjects were also informed that these electrodes, which were used to record their brain activity, would not do them any physical harm. A 2 (male/female) $\quad$ x 2 (humorous/non-humorous ads) factorial design was employed. Eight subjects were randomly assigned to each cell, with the exception that nine females were randomly assigned to the cell of watching the humorous advertisement.

\subsection{Data collection and experimental environment}

The social media advertisement and the computer system used in the experiment were designed explicitly for this study. To avoid potential confounding biases, the experiment was conducted in a controlled laboratory setting. Two identical personal computers with 24" LCD monitors were set up in a laboratory. Each computer was pre-assigned to run one of the two treatments. Internet Explorer 9.0 was set as the constant Internet browser for all the subjects.

The data collection process employed a brain activity monitoring system (model name: EEG2000) using four electrodes to acquire high resolution neurological signals. Two electrodes, located just behind the participants' ears (one at each side), were used as references. A further two electrodes were located on the subject's forehead to collect signals from frontal brain aspects. The system software controlled the recording process to ensure that no data were lost, and a real-time electrode connection display ensured the quality of the measurements.

\subsection{Experiment task and procedures}

In this experiment, subjects were assigned a task of watching a social media advertisement for a new company. To avoid the possible biased impression that subjects might have had toward an existing or familiar brand name, a new company that had been established six months prior to this study was chosen as the experiment target. This new company focused on the social business of promoting children's food education.

For the research purpose, a baseline film and the social media advertising must be designed. As the biological neural status of each participant is different, it is crucial to record the initial four brain powers of each subject to build the index level. A pilot study was conducted upon the design of the baseline film. The first design was a static earth picture lasting for one minute. The second design was white clouds in the sky moving slowly towards the subject for one minute. Ten volunteer participants watched the 1 st version, and another ten watched the 2 nd one. Their neural data recorded while they were watching these films showed that the first design was not suitable, as the subjects may have become sleepy, as higher delta and theta brain 
powers were found. Consequently, the cloud film was selected.

Two versions of the advertisement designed to promote brand awareness were produced explicitly for this study. The core contents of these two versions were the same. The humor manipulation was conducted by adding a humorous comic and adjectives in one version, while the non-humorous advertisement remained as a plain description.

After the electrodes had been installed, the respondents started the experiment, which had two stages. In the 1st stage of the experiment, each subject was required to watch a baseline film for one minute. In this film, white clouds in the sky move slowly towards the subject. The "EEG baseline" factor was recorded at this stage.

In the 2nd stage, the subjects were randomly assigned to watch one of the two versions of the advertisement playing on an online social media channel. The only difference was one version had additional funny animations while the other did not. The "EEG watching ad" factor was recorded at this stage. The film contents of these two versions were the same.

Upon completing the experiment, each subject was offered a reward valued at around US\$ 3.3 dollars as a mark of appreciation for their full participation and efforts.

\section{Using fsQCA for Model Evaluation 4.1 Set-membership measures}

The sum and accumulated percentage of EEG values were calculated so as to set the anchors more appropriately while calibrating the variables. Three anchors were set as 95\%, 50\%, and 5\%. The EEG brain activity power was thus transformed into the corresponding data within $0 \sim 1$ and generated eight values: left frontal delta power (ld), left frontal theta power (lt), left frontal alpha power (la), left frontal beta power (lb), right frontal delta power (rd), right frontal theta power (rt), right frontal alpha power (ra), and right frontal beta power $(\mathrm{rb})$. Attention towards social media advertisements was also calibrated using the same rule.

Specifically, to further mark the different neural data collected during the two experimental stages, that is, "EEG baseline" factor and "EEG watching advertising" factor, four brain powers of frontal power collected during the former stage are labeled as "ld.base", "lt.base", "la.base", and "lb.base", respectively. Similarly, the four brain powers of frontal power collected during the latter stage are labeled as "ld.ad", "lt.ad", "la.ad", and "lb.ad", respectively. The neural data collected from the right frontal brain is labeled using the same rule.
Male/female and advertisement type were calibrated as 1 for male and 0 for female, and 1 for the humorous advertisement, 0 for the non-humorous advertisement.

\subsection{Consistency in set relations}

Consistency is a useful index while seeking configurations that are sufficient conditions for consumers' neurological responses. The consistency value can be obtained in an XY plot or by conducting a truth table analysis. A consistency value over 0.8 is considered as an acceptable level. This study proposed one model to test the effects of left frontal brain activity, including EEG at baseline and EEG while watching the advertisement, and one model to test the effects of right frontal brain activity. Figure 4, below, shows the results of the analysis for these two models respectively. Specifically, the left frontal model reported a consistency of 0.86 and coverage of 0.14 using the XY plot, and a consistency of 0.93 and coverage of 0.66 using the truth table analysis. The right frontal model indicated a consistency of 0.87 and coverage of 0.14 using the XY plot, and a consistency of 0.95 and coverage of 0.54 using the truth table analysis. Both models reported acceptable levels of consistency. Notably, as gender and advertisement type were the variables manipulated in this study, only formulas including both gender and advertisement type were considered in the testing procedures below: i.e., only five receipts in the left frontal model and all thirteen receipts in the right frontal model.

\subsection{Propositions Testing}

A simplified procedure further uncovered that there were three groups within the left frontal brain activity model: male/humorous advertisement (formula 8, 11, 12); female/humorous advertisement (formula 10); and male/non-humorous advertisement (formula 8, 11, 13). Formula 10 revealed that a receipt of female, watching a humorous advertisement, gave a baseline EEG reading of a high level of alpha, theta, and delta power, and an EEG while watching the advertisement revealed high levels of all four brain powers. In contrast, formula 11, for example, revealed that a receipt of male, watching a humorous advertisement, gave a baseline EEG reading of a high level of alpha, theta, and delta power, but an EEG while watching the advertisement revealed low levels of beta, theta, and alpha power, with only the delta power being high. That is, for the same humorous advertisement, women and men displayed diverse EEG brain activity receipts. Further, formula 13 revealed that a receipt of male, watching a non-humorous advertisement, gave a baseline EEG reading a high level of all four bands (i.e., beta, alpha, theta, and delta power), 
but an EEG while watching the advertisement revealed high levels of beta, theta, and alpha power, with only the delta power being low. When comparing the results of formulae 10, 11, and 13, P2 was supported. Moreover, the consistency (0.93) and coverage (0.66) level of the left frontal model showed adequate support for P1 and P4. Thus, P1 and P4 were confirmed.
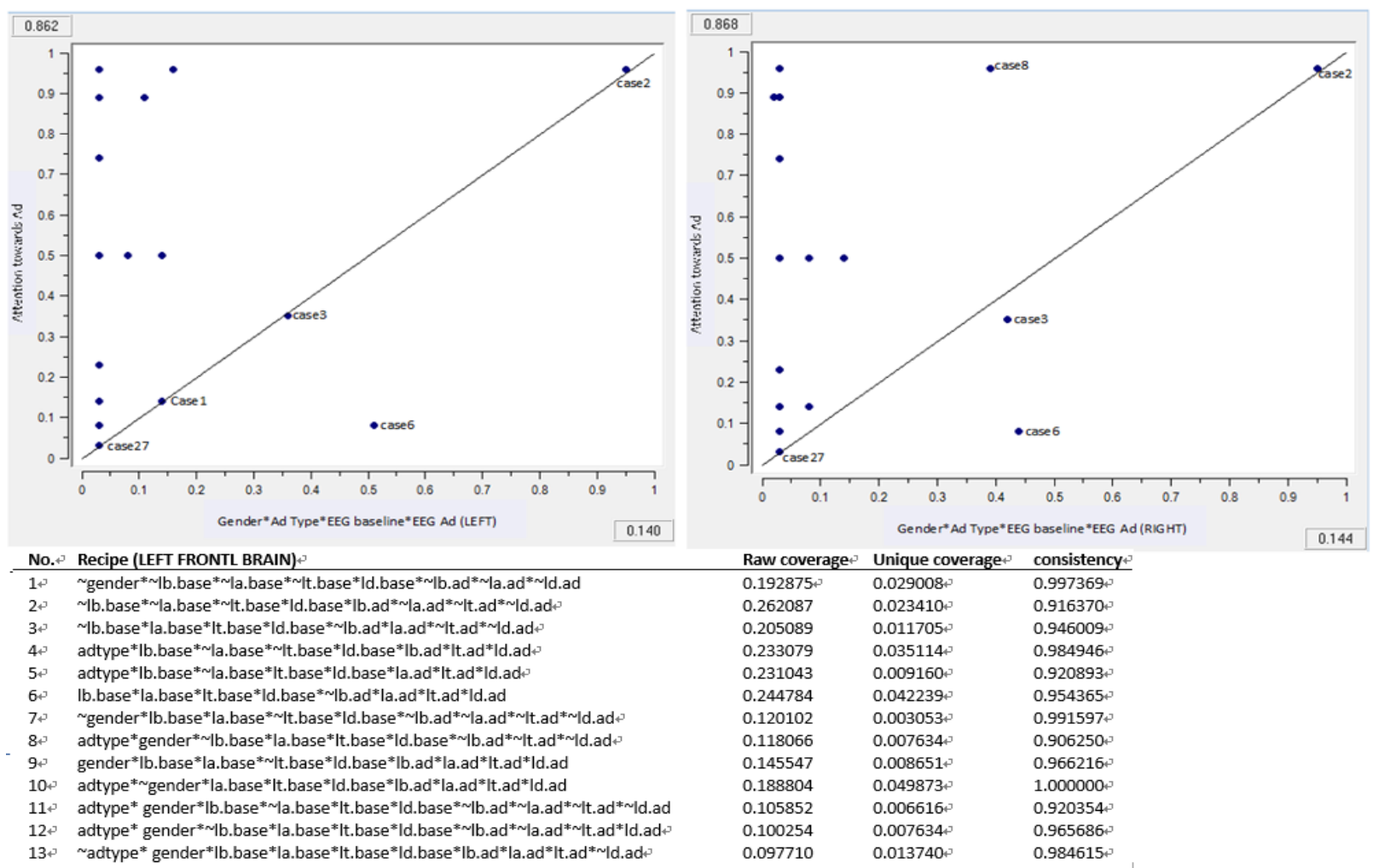

\begin{tabular}{|c|c|}
\hline & 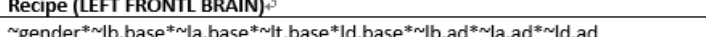 \\
\hline 2 & 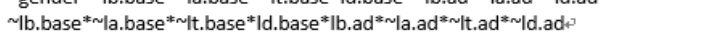 \\
\hline 3 & 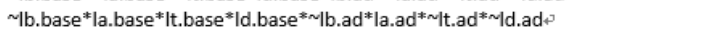 \\
\hline 4 & 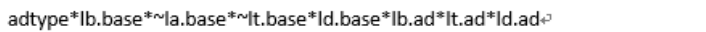 \\
\hline 5 & 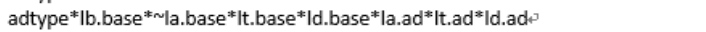 \\
\hline 6 & |b.base*|a.base ${ }^{*} \mid$ t.base*|d.base ${ }^{*} \mid$ |b.ad*la.ad*|t.ad*Id.ad \\
\hline 7 & 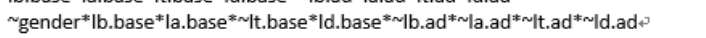 \\
\hline 8 & 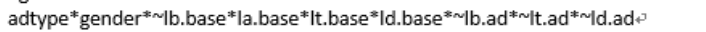 \\
\hline 9 & gender*|b.base*|a.base*n|t.base*|d.base*|b.ad*la.ad*|t.ad*Id.ad \\
\hline 10 & 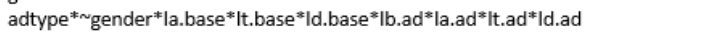 \\
\hline 11 & 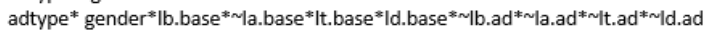 \\
\hline 12 & 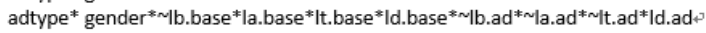 \\
\hline 13 & 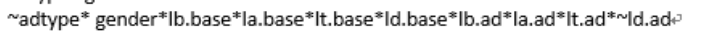 \\
\hline & on consistency: 0.933381 \\
\hline
\end{tabular}

\begin{tabular}{|c|c|c|c|c|}
\hline No. & Recipe (RIGHT FRONTL BRAIN) & Raw coverage & Unique coverage & consistency \\
\hline $1+$ & 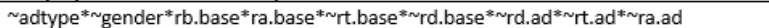 & 0.128753 & 0.036641 & 0.913357 \\
\hline 2 & adtype $^{*}$ gender*rb.base*ra.base ${ }^{*}$ rd.base* rd.ad*rt.ad*ra.ad*rb.ad & 0.214249 & 0.002036 & 1.000000 \\
\hline 3 & adtype* ${ }^{*}$ gender ${ }^{*}$ rb. base $e^{*}$ rt.base* rd.base* rd.ad* rt.ad*ra.ad*rb.ad & 0.217812 & 0.002544 & 1.000000 \\
\hline 4 & 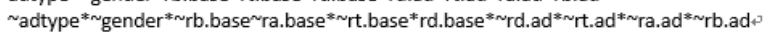 & 0.099746 & 0.009160 & 0.956098 \\
\hline 5 & $\sim$ adtype ${ }^{* \sim}$ gender $^{* \sim}$ rb.base $e^{* \sim}$ ra.base $e^{* \sim}$ rt.base $e^{* \sim}$ rd. base ${ }^{* \sim}$ rd.ad ${ }^{*}$ rt.ad ${ }^{* \sim}$ ra.ad $d^{* \sim}$ rb.ad & 0.109924 & 0.004071 & 0.990826 \\
\hline 6 & 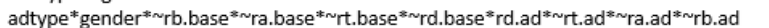 & 0.079898 & 0.023410 & 0.934524 \\
\hline 7 & 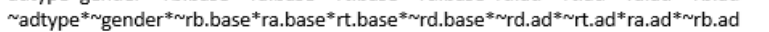 & 0.100254 & 0.019338 & 0.989950 \\
\hline 8 & 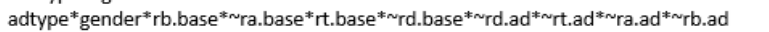 & 0.087532 & 0.017812 & 0.934783 \\
\hline 9 & 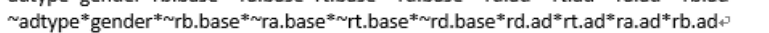 & 0.096692 & 0.010178 & 0.954774 \\
\hline 10 & adtype*gender ${ }^{*}$ rb.base ${ }^{*}$ ra.base $e^{*} \sim$ rt.base ${ }^{*} \sim$ rd. base* ${ }^{*}$ rd.ad $d^{*} \sim$ rt.ad ${ }^{*}$ ra. ad ${ }^{*} \sim$ rb.ad & 0.079898 & 0.008142 & 0.934524 \\
\hline 11 & 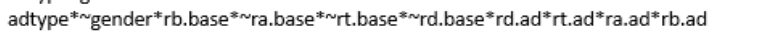 & 0.147583 & 0.008142 & 1.000000 \\
\hline 12 & $\sim_{\text {adtype }}^{*}$ gender* ${ }^{*}$ rb.base ${ }^{*}$ ra.base ${ }^{*}$ rt.base ${ }^{*} \sim$ rd.base ${ }^{*}$ rd.ad ${ }^{*}$ rt.ad ${ }^{*}$ ra.ad ${ }^{*}$ rb.ad & 0.097201 & 0.010178 & 0.959799 \\
\hline soluti & $\begin{array}{l}\text { adtype*gender }{ }^{*} \text { rb.base } e^{*} \text { ra.base* rt.base }{ }^{*} \text { rd.base }{ }^{*} \sim \text { rd. }^{*} d^{*} \text { rt.ad } \\
\text { ion coverage: } 0.548600\end{array}$ & 0.114504 & 0.036641 & 0.941423 \\
\hline
\end{tabular}

Figure 4. XY plot and truth table analysis results

Regarding the right frontal brain activity model, there are four different groups: male/humorous advertisement (formulae 6, 8, 10, and 13); female/humorous advertisement (formulae 2, 3, and 11); male/non-humorous advertisement (formulas 9 , 12, and 13); and female/non-humorous advertisement (formulae 1, 4, 5, and 7). Formula 3 reported that a receipt of female, watching a humorous advertisement, gave a baseline EEG reading of a high level of beta, theta, and delta power, and an EEG while watching the advertisement revealed high levels of all four brain powers. In contrast, formula 4 , for example, revealed a receipt of female, watching a non-humorous advertisement, gave a baseline EEG reading of a high level of beta and delta power, with a low level of alpha and theta power. Additionally, an EEG while watching the advertisement revealed low levels of all 
four brain powers. That is, for the same gender (female), the brain activity displayed diverse EEG brain activity while watching humorous/nonhumorous advertisements. Further, formula 8 revealed that a receipt of male, watching a humorous advertisement, gave a baseline EEG reading of a high level of beta and theta power with a low level of alpha and delta power. Further, an EEG while watching the advertisement reported a low level of all four brain powers. Last, formula 9 revealed that a receipt of male, watching a non-humorous advertisement gave a baseline EEG readings of low level for all four bands, but an EEG while watching the advertisement revealed high levels of all four brain powers. By contrasting the results of formulae 3, 4, 8, and 9, P2 was supported. Moreover, the consistency (0.95) and coverage $(0.55)$ levels of the right frontal model showed adequate support for P1, P3 and P4. Thus, P1, P3 and P4 were also confirmed.

\section{Conclusion}

Based on the discussion presented in sections 2 and 4, Table 1, below, reports the findings provided by fsQCA in relation to the major research questions of this study.

Table 1. Findings provided by fsQCA

\begin{tabular}{|l|l|}
\hline \multicolumn{1}{|c|}{ Research Question } & $\begin{array}{c}\text { Results provided by } \\
\text { FsQCA }\end{array}$ \\
\hline $\begin{array}{l}\text { Do men and women } \\
\text { differ in their EEG brain } \\
\text { activity while watching } \\
\text { humorous/non-humorous } \\
\text { advertisements? }\end{array}$ & $\begin{array}{l}\text { YES. More } \\
\text { specifically, the right } \\
\text { frontal brain reported } \\
\text { more significant } \\
\text { results than the left } \\
\text { frontal brain. }\end{array}$ \\
\hline $\begin{array}{l}\text { Can consumers' EEG } \\
\text { brain activity while } \\
\text { watching social media } \\
\text { advertisement predict } \\
\text { their attention towards } \\
\text { social media } \\
\text { advertisements? }\end{array}$ & $\begin{array}{l}\text { YES. The EEG brain } \\
\text { activity while } \\
\text { watching social media } \\
\text { advertisements was a } \\
\text { useful predictor of } \\
\text { consumers' attention. }\end{array}$ \\
\hline $\begin{array}{l}\text { Will the EEG baseline } \\
\text { brain activity help to } \\
\text { better explain the } \\
\text { research model? }\end{array}$ & $\begin{array}{l}\text { YES. The EEG } \\
\text { baseline brain activity } \\
\text { increased the model's } \\
\text { consistency value. }\end{array}$ \\
\hline
\end{tabular}

Empirical findings provided using the fsQCA method have confirmed the research propositions: men and women reported diverse EEG brain activity receipts while watching the social media advertisement; men and women displayed different EEG brain activity receipts towards humorous/non- humorous social media advertisement; and consumers' EEG brain activity receipt (including EEG baseline and EEG while watching the advertisement) was able to predict their levels of attention towards the social media advertisement. Overall, the results offered by the fsQCA method demonstrated good support for this study's research questions.

The current study has added value to the research field in three ways. First, it is likely to be the first study to reveal empirically how men and women differ in their response to humorous social media advertisements from a neuroscience perspective. More importantly, while past neuromarketing studies have tended to involve samples of fewer than ten participants due to the inherent difficulties in collecting brain activity data from subjects (such as the high cost of the machine and people's reluctance to participate because of the physical discomfort of wearing sensors), the current study has successfully collected data from 33 participants, which has greatly enhanced the generalizability of the findings. Second, this study has provided evidence of how EEG brain activity might be used to predict consumers' attention towards social media advertisements. Third, by demonstrating how the fsQCA method could be used to reveal consumers' EEG brain activity, this study's contribution has offered a good example for the neuroscience research discipline.

This study's findings are also useful for social media advertisements pre-testing. To create one successful social media advertisement, several pretesting advertisements - sometimes even dozens might be necessary. Past studies, which often applied interview or questionnaire methods to collect consumers' responses toward the pretesting advertisement, have shown great concern about the biased results created by audiences who hide their true feelings. By demonstrating that men and women perceive humor communications applied in social media advertisements differently and how EEG brain activity can be used to evaluate consumers' attention towards a social media advertisement, this study's findings help practitioners not only in building marketing segmentation and product/brand positioning strategies, but also in how to use an innovative evaluation technique for advertisements communicating effectiveness so as to understand better their target customers' responses. To provide more insights, future studies are suggested to validate the current study's findings in different product categories such as mobile phones or luxury handbags.

This study has some limitations. First, we have not yet investigated the EEG brain activity receipt in a more extensive way. This discussion might be developed in another study. Second, caution should 
be exercised while applying the findings of this study, which were based on thirty-three individuals who volunteered to participate in the experiment. Nonetheless, there is a need for future neuromarketing studies to test brain activity data continuously using the fsQCA method to further verify its potential in offering adequate explanations of the research data.

\section{References}

[1] Apte, M. L., "Humor and laughter: An anthropological approach", Ithaca, New York: Cornell University Press, 1985.

[2] L. Astolfi, F.D.V. Fallani, F. Cincotti, D. Mattia, L. Bianchi, M.G. Marciani et al. "Neural basis for brain responses to TV commercials: A high-resolution EEG study", IEEE Transactions on Neural Systems and Rehabilitation Engineering, 2008, 27(6), pp.522-531.

[3] S.B. Barnett, \& M. Cerf, "Connecting on movie night? Neural measures of engagement differ by gender", Advances in Consumer Research, 2015, 43, pp. 314-318.

[4] T.E. Barry, \& D.J. Howard, "A review and critique of the hierarchy of effects in advertising", International Journal of Advertising, 1990, 9(2), pp. 98-111.

[5] S.L. Bem, "Gender schema theory: A cognitive account of sex typing", Psychological Review, 1981, 88(4), pp. 354364 .

[6] L. Cahill, "Why sex matters for neuroscience", Nature Reviews Neuroscience, 2006, 7(6), pp. 477-484.

[7] T.K.H. Chan, C.H. Cheung, N. Shi, \& M.K.O. Lee, "Gender differences in satisfaction with Facebook users", Industrial Management \& Data Systems, 2015, 115(1), pp182-206.

[8] S.C. Chu, "Viral advertising in social media: Participation in Facebook groups and responses among college-aged users", Journal of Interactive Advertising, 2013, 12(1), pp30-43.

[9] K.P. Cosgrove, C.M. Mazure, \& J.K. Staley, "Evolving knowledge of sex differences in brain structure, function, and chemistry", Biological Psychiatry, 2007, 62(8), pp. 847855 .

[10] T. Costa, E. Rognoni, \& D. Galati, "EEG phase synchronization during emotional response to positive and negative film stimuli”, Neuroscience Letters, 2006, 406, pp. 159-164.

[11] S. Coulson, \& M. Kutas, "Getting it: Human eventrelated brain response to jokes in good and poor comprehenders", Neuroscience Letters, 2001, 316, pp. 7174.

[12] S.E. Cross, \& L. Madson, "Models of the self: Selfconstruals and gender", Psychological Bulletin, 1997, 122, pp. 5-37.
[13] V.T. Dao, N.H. Le, M.S. Cheng, \& D.C. Chen, "Social media advertising value: The case of transitional economies in Southeast Asia", International Journal of Advertising, 2014, 33(2), pp. 271-294.

[14] W.K. Darley, \& R.E. Smith, "Gender differences in information processing strategies: An empirical test of the selectivity model in advertising response", Journal of Advertising, 1995, 24(1), pp. 41-56.

[15] Darwin, C., "The Descent of Man”, London: Murray, 1871.

[16] A. Dennis, S. Kinney, \& Y. Hung, "Gender differences in the effects of media richness", Small Group Research, 1999, 30(4), pp. 405-438.

[17] S. Gabriel, \& W.L. Gardner, "Are there 'his' and 'hers' types of interdependence? The implications of gender differences in collective versus relational interdependence for affect, behavior, and cognition", Journal of Personality and Social Psychology, 1999, 77, pp. 642-655.

[18] D. Gefen, \& D.W. Straub, "Gender differences in the perception and use of e-mail: An extension to the technology acceptance model", Mis Quarterly, 1997, 21(4), pp. 389-400.

[19] G. Greengross, \& G. Miller, "Humor ability reveals intelligence, predicts mating success, and is higher in males", Intelligence, 2011, 39(4), pp. 188-192.

[20] D.P. Howrigan, \& K.B. MacDonald, "Humor as a mental fitness indicator", Evolutionary Psychology, 2008, 6, pp. 652-666.

[21] J. Imlawi, \& D. Gregg, "Engagement in online social networks: The impact of self-disclosure and humor", International Journal of Human-Computer Interaction, 2014, 30(2), pp. 106-125.

[22] S.A. Inks, S. Schetzsle, \& R.A. Avila, "Exploring the use of business social networking tools in sales: Current perceptions and future expectation”, Marketing Management Journal, 2012, 22(1), pp. 1-16.

[23] S. Jhally, The Codes of Advertising, 1987, New York, NY: St. Martin's Press.

[24] K. Judson, R. Devasagayam, \& C. Buff, "Self-perceived brand relevance of and satisfaction with social media", Marketing Management Journal, 2012, 22(2), pp. 131-144.

[25] R. Khushaba, C. Wise, S. Kodagoda, J. Louviere, B.E. Kahn, \& C. Townsend, "Consumer neuroscience: Assessing the brain response to marketing stimuli using electroencephalogram (EEG) and eye tracking", Expert Systems with Applications, 2013, 40, pp. 3803-3812.

[26] M. Laroche, M. Nepomuceno, R. V. Marie-Odile, "Congruency of humour and cultural values in print ads", International Journal of Advertising, 2014, 33(4), pp681-705.

[27] R.J. Lavidge, \& G.S. Steiner, "A model for predictive measurements of advertising effectiveness", Journal of Marketing, 1961, 25(4), pp. 59-62.

[28] E. Liew, S. Vaithilingam, \& N. Mahendhiran, "Facebook and socio-economic benefits in the developing 
world", Behaviour \& Information Technology, 2014, 33(4), pp. 345-360.

[29] J.S. Lim, K.S Lim, \& J.H. Heinrichs, "Gender and mobile access method differences of millennials in social media evaluation and usage: An empirical test", Marketing Management Journal, 2014, 24(2), pp124-135.

[30] Y. Lin, C. Wang, T. Jung, T. Wu, S. Jemg, J. Duann, \& J. Chen, "EEG-based emotion recognition in music listening", IEEE Transactions on Biomedical Engineering, 2010, 57(7), pp. 1798-1806.

[31] D.E. Lundy, J. Tan, \& M. Cunningham, "Heterosexual romantic preferences: The importance of humor and physical attractiveness for different types of relationships", Personal Relationships, 1998, 5(3), pp. 311-325.

[32] A.K.Y. Mak, Z. Hu, J.X.X. Zhang, Z. Xiao, \& T.M.C. Lee, "Sex-related differences in neural activity during emotion regulation", Neuropsychologia, 2009, 47(13), pp. 2900-2908.

[33] P. McGraw, C. Warren, \& C. Kan "Humorous complaining", Journal of Consumer Research, 2015, 41(5), pp. 1153-1171.

[34] V. Melnyk, S.M.J. Osselaer, \& T.H.A. Bijmolt, “Are women more loyal customers then men? Gender differences in loyal to firms and individual service providers", Journal of Marketing, 2009, 73(4), pp. 82-96.

[35] D. Nie, X.W. Wang, L.C. Shi, \& B.L. Lu, "EEG-based emotion recognition during watching movies", The 5th international IEEE EMBS conference on neural engineering, Cancun, Mexico, April 27-May 1, 2011.

[36] R. Ohme, D. Reykowska, D. Wiener, \& A. Choromanska, "Application of frontal EEG asymmetry to advertising research", Journal of Economic Psychology, 2010, 31(5), pp. 785-793.

[37] Panksepp, J., "Neurochemical control of moods and emotions: Amino acids to neuropeptides" In M. Lewis, \& J. Haviland (Eds.), The handbook of emotions, (pp. 87-107). New York: Guilford, 1993.

[38] R. Pieters, M. Wedel, \& R. Batra, "The Stopping Power of Advertising: Measures and Effects of Visual Complexity", Journal of Marketing, 2010, 74 (5), pp 48-60.

[39] E. Porter, N. Donthu, \& A. Baker, "Gender differences in trust formation in virtual communities", Journal of Marketing Theory \& Practice, 2012, 20(1), pp. 39-58.

[40] R. Riedl, M. Hubert, \& P. Kenning, "Are there neural gender differences in online trust? An fMRI study on the perceived trustworhiness of ebay offers", MIS Quarterly, 2010, 34(2), pp. 397-428.

[41] J.P. Rushton, \& C.D. Ankney, "Brain size and cognitive ability: Correlations with age, sex, social class, and race", Psychonomic Bulletin \& Review, 1996, 3(1), pp. 21-36.

[42] M. Shibata, Y. Terasawa, \& S. Umeda, "Integration of cognitive and affective networks in humor comprehension", Neuropsychologia, 65, pp. 137-45.
[43] D.H. Shin, "User experience in social commerce: in friends we trust", Behaviour \& Information Technology, 2013, 32(1), pp52-67.

[44] N. Shirao, Y. Okamoto, T. Mantani, Y. Okamoto, \& S. Yamawaki, "Gender differences in brain activity generated by unpleasant word stimuli concerning body image: An fMRI study", British Journal of Psychiatry, 2005, 186(1), pp. 48-53.

[45] E.K. Strong, "Theories of Selling", The Journal of Applied Psychology, 1925, 9(1), pp. 75-86.

[46] G. Vecchiato, W. Kong, A.G. Maglione, \& D. Wei, "Understanding the impact of TV commercials", IEEE Pulse Magazine, 2012, 3(3), pp. 42-47.

[47] A. Yoto, T. Katsuura, K. Iwanaga \& Y. Shimomura, "Effects of object color stimuli on human brain activities in perception and attention referred to EEG alpha band response", Journal of Physiological Anthropology, 2007, 26(3), pp. 373-379

[48] L.T. Zayer \& C.A. Coleman, “Advertising professionals' perceptions of the impact of gender portrayals on men and women: A question of ethics?", Journal of Advertising, 2015, 44 (3), pp.1-12.

[49] K.Z.K. Zhang, C.M.K. Cheung, \& M.K.O. Lee, "Examining the moderating effect of inconsistent reviews and its gender differences on consumers' online shopping decision", International Journal of Information Management, 2014, 34(2), pp. 89-98.

[50] K.Z.K. Zhang, M.K.O. Lee, C.M.K. Cheung, \& H. Chen, 'Understanding the role of gender in bloggers' switching behavior", Decision Support Systems, 2009, 47(4), pp. 540-546. 